\title{
THE ISIS SYNCHROTRON BEAM CONTROL AND STUDY PROGRAMME
}

\author{
D.J.Adams, K.Tilley, C.M.Warsop, Rutherford Appleton Laboratory, Oxfordshire, UK.
}

\begin{abstract}
Progress on the beam control and study programme for the $800 \mathrm{MeV}$ High Intensity Proton Synchrotron of the Spallation Neutron Source ISIS, is outlined. Recent hardware upgrades to diagnostics, instrumentation and computing have increased the amount, accuracy and availability of beam information. The measurement methods employed and their planned applications for beam control, optimisation and study are described. Work includes detailed study of longitudinal and transverse dynamics at high and low intensity. Results obtained so far and future plans are summarised.
\end{abstract}

\section{INTRODUCTION}

\subsection{Background}

This paper summarises progress on the beam control and study programme, which was detailed in an earlier paper [1]. Here, recent experimental results from the upgraded hardware are summarised, along with their application to machine control.

The ISIS Synchrotron [2] accelerates $2.5 \times 10^{13}$ protons per pulse at $50 \mathrm{~Hz}(200 \mu \mathrm{A})$. The high intensity beam is established via charge exchange injection over 120 turns. Beam is then bunched and accelerated from $70-800 \mathrm{MeV}$ in $10 \mathrm{~ms}$, extracted in a single turn and transported to the target.

Extensive use is made of low intensity 'diagnostic' beams [1]. These provide additional, detailed information which complements high intensity measurement. On ISIS suitable beams are produced by 'chopping' the injection pulse from the normal 120 turns to less than 1 turn. Such beams can be interleaved with normal $50 \mathrm{~Hz}$ high intensity pulses; typical 'chopping' at 1 pulse in 128 has minimal effect on operational beam.

\subsection{Synchrotron Diagnostics Upgrades}

The diagnostics hardware upgrade is based on the addition of many fast digitising channels to make full use of the existing instrumentation. Twenty $100 \mathrm{MS} / \mathrm{s}$ digitisers allow turn by turn beam position measurements at 10 monitors simultaneously. Two $1 \mathrm{GS} / \mathrm{s}$ digitisers allow the acquisition of longitudinal profiles. Position monitor electronics upgrades enable measurements with both high and low intensity beams.

A dedicated DEC Alpha work station linked to the control system allows extensive hardware control, thus facilitating automated measurement. The increased computing power enables the use of high level data analysis software and graphical user interfaces, which simplify correction procedures significantly.

\section{CLOSED ORBIT MEASUREMENT AND CORRECTION}

\subsection{Closed Orbit Correction and Lattice Model}

Closed orbit data is now readily acquired and processed on the new systems. Closed orbit correction and manipulation is based on the solution to the linear equation:

$$
x_{i}=A_{i j} \cdot b_{j}
$$

The elements of steering matrix $\boldsymbol{A}_{\mathrm{ij}}$ give the closed orbit deviation $\mathbf{x}_{\mathrm{i}}$ at monitor $\mathrm{i}$ due to an angular kick $\boldsymbol{b}_{\mathrm{j}}$ at corrector j. Optimal corrections are found by reducing $\boldsymbol{A}_{\mathrm{ij}}$ using Singular Value Decomposition and solving Eq.1 for $\boldsymbol{b}_{\mathrm{j}}$.

A synchrotron lattice model has been developed to calculate linear lattice parameters and track particle trajectories. The model is very effective for manipulation and correction of closed orbits, by generating the steering matrix $\boldsymbol{A}_{\mathrm{ij}}$ and solving Eq.1.

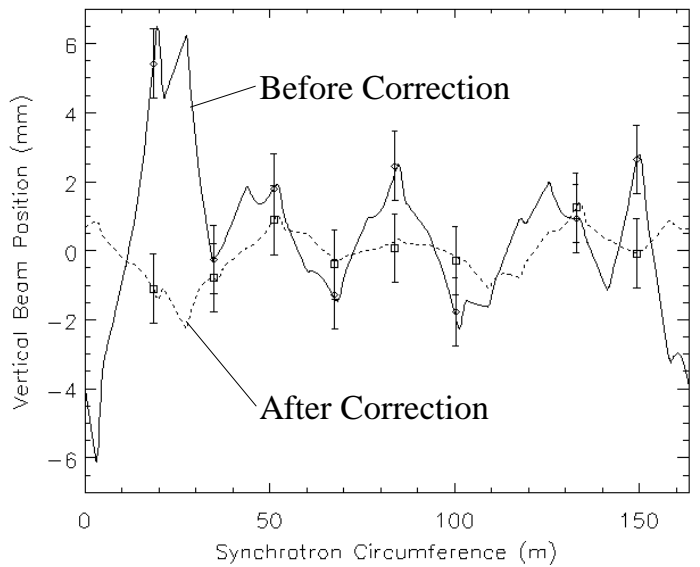

Figure 1: Vertical C.O. measured before and after correction, with modelled trajectory.

Figure 1 shows an example of the measured high intensity vertical closed orbit before and after correction with the lattice model. The model optimises kicks at 7 steering magnets, to fit measured closed orbit errors. As would be expected from the well optimised set of 
correctors, calculated orbits can be seen to fit measurements very well ( $\mathrm{rms}$ difference $<1 \mathrm{~mm}$ ). Application of the calculated kicks typically reduces the peak closed orbit errors from $\sim 6 \mathrm{~mm}$ to $\sim 1 \mathrm{~mm}$ in three iterations. The correction could be improved further by using a measured steering matrix, which will be possible when automated magnet control becomes available.

The correction technique has now been incorporated in the machine set-up schedule. The time to correct, or reestablish empirically optimised 'Golden Orbits', throughout acceleration has been reduced from days to hours. Planned automation should reduce this to minutes.

\subsection{Further Closed Orbit Work}

Correction of closed orbits at injection will be much improved with the use of chopped beams, where AC coupled monitors and the unbunched beam prevents reliable high intensity orbit measurements. More sophisticated uses of the measurements and models are envisaged, including the location of alignment and field errors. Good models of beam trajectories and closed orbits, at high and low intensity, will be useful for aperture studies and beam loss collector set-up.

\section{BETA FUNCTION}

\subsection{Multi-turn Position Measurements}

Measurement of turn by turn, coherent transverse motion at a given monitor allows numerous ring parameters to be extracted by fitting a suitable function [3]. Most important are centroid betatron amplitude (b), closed orbit, betatron $\mathrm{Q}$ and phase. Measurements are possible at high and low intensity but the most accurate results come from the latter. Here low intensity measurements at injection are presented; the injection painting process conveniently provides the coherent motion. Future developments will allow measurements throughout the machine cycle, using a fast kicker to excite the beam.

Multiple digitisers and suitable monitors now allow simultaneous measurement of the above parameters at 10 points around the ring. This allows reconstruction of closed orbit, phase advance and beta functions. Below, first measurements on beta functions are summarised.

\subsection{Beta Function Measurements}

The betatron amplitude $\left(b_{\mathrm{i}}\right)$ at $\sim 10$ monitors is measured on the same pulse. The relation to the local beta function $\left(\beta_{\mathrm{i}}\right)$ is $\mathrm{b}_{\mathrm{i}}=\sqrt{ }\left(\beta_{\mathrm{i}} \varepsilon_{\mathrm{c}}\right)$, where the centroid emittance $\left(\varepsilon_{\mathrm{c}}\right)$ is identical at all monitors for a given pulse. In comparing measurements sets, variation of $\varepsilon_{\mathrm{c}}$ from one pulse to another has to be accounted for.

To demonstrate the validity of the basic measurements a set of $b_{i}$ were measured first with the synchrotron in a normal configuration, and then with a deliberate gradient error introduced by switching off one trim quadrupole. The theoretical change in the beta function due to such perturbations is known and gives an envelope oscillation at $2 \mathrm{Q}$, given by the following formula:

$\frac{\Delta \beta(s)}{\beta(s)}=\mp \frac{1}{2 \sin 2 \pi Q} \oint \Delta k(\sigma) \beta(\sigma) \cos 2[|\mu(\sigma)-\mu(s)|-\pi Q] d \sigma$

where the notation is standard [4]. The data was then brought into the above form using both measurement sets, and the optimal least squares fit determined. To simplify interpretation, only measurements at equivalent lattice positions in the 10 superperiods (where the $\beta_{\mathrm{i}}$ are ideally identical) were considered. The results are plotted in Figure 2.

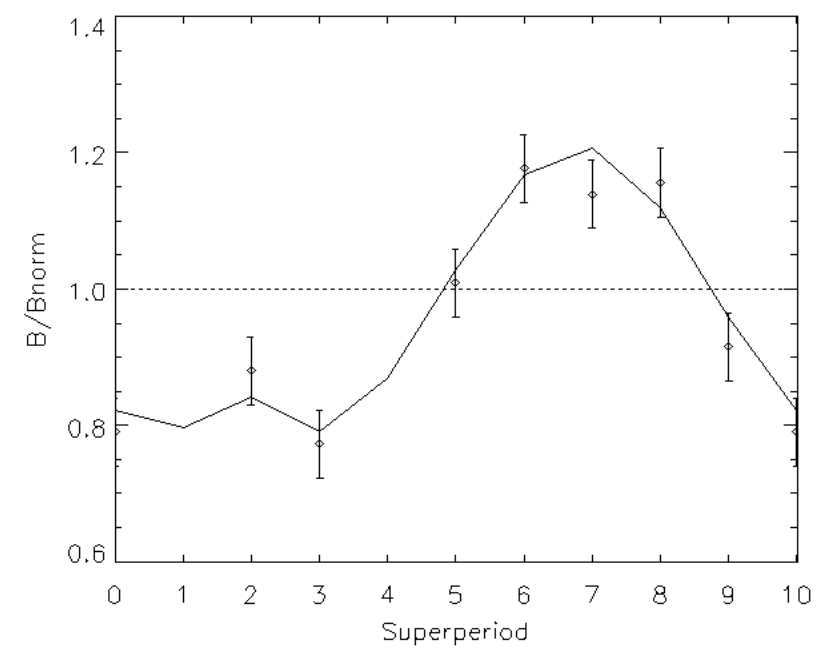

Figure 2: Perturbation in Relative Beta due to Gradient Error in Superperiod 2; Measurement and Theory.

Agreement between the functional forms is good and gives confidence in the measurements. A fuller theoretical comparison is being developed.

\subsection{Further Applications}

An obvious application for these measurements is to correct beta function perturbation by applying appropriate harmonics to the 20 trim quadrupoles. Measurements could also be used to locate single large gradient errors. Use of two monitors separated by a drift space, to deduce $\left(\mathrm{y}, \mathrm{y}^{\prime}\right)$ on a turn by turn basis, should allow measurement of absolute beta at one monitor; this combined with the above provides beta at all monitors. Absolute beta at each trim quad can also be calculated by measuring the change in $\mathrm{Q}$ as a function of quadrupole current [1]. Collectively these measurements would yield the beta function at 30 points per plane. 


\section{LONGITUDINAL MEASUREMENTS}

\subsection{Basic Measurements}

These measurements are based on digitising the longitudinal bunch shapes from a capacitative monitor over thousands of turns. Some processing, making use of the digitised RF voltage, yields the instantaneous beam intensity as a function of RF phase on every turn. Moments analysis of the turn by turn longitudinal distributions then provides the bunch centroid phase and bunch length on each turn. Using these techniques the dynamics of both high and low intensity beams can be studied. Early results are shown below.

\subsection{High and Low Intensity Measurements}

Examples of bunch length measurements for a high intensity beam are given in Figure 3. This shows bunch lengths at various percentages of the peak instantaneous current. The $95 \%$ contour follows the theoretical (zero intensity) RF bucket length in the expected manner, with high bucket occupancy during trapping which then decreases through acceleration. Oscillation at the synchrotron frequency is also visible.

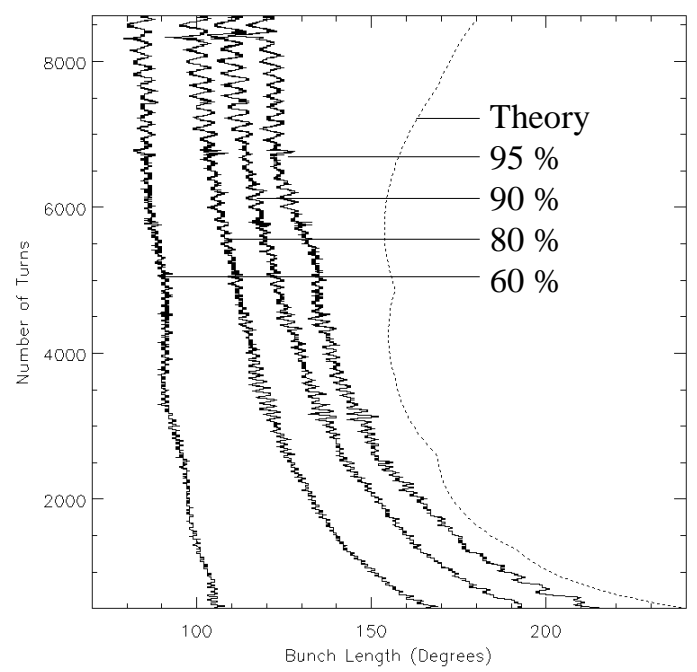

Figure 3: High Intensity Bunch Length Measurement compared with theoretical RF Bucket Length.

The injection and acceleration of chopped beams, occupying less than one turn, reveal details of longitudinal motion. Depending on the bunch length, and relative phase of the injected beam with respect to the RF, dipole and bunch length oscillations of various amplitudes can be set up. From this, using the processing described above, the FFT of first and second moments of the longitudinal distribution over thousands of turns allows extraction of the synchrotron tune $\mathrm{Q}_{\mathrm{s}}$. Figure 4 shows good agreement with theory.

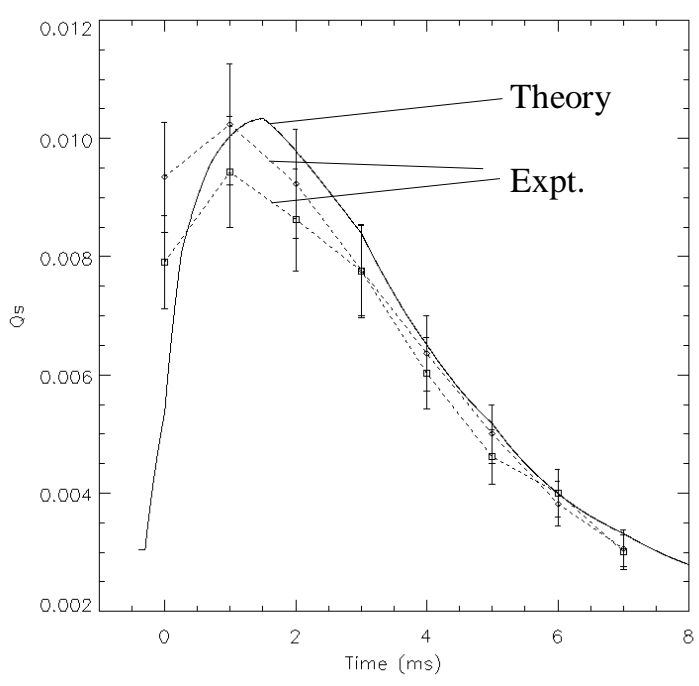

Figure 4: Synchrotron Tune $\left(Q_{s}\right)$ through the machine cycle; Low Intensity Measurements and Theory.

\subsection{Further Studies}

The above results represent the first stage of measurement development. More sophisticated studies are planned. These will include detailed investigation of phase stable regions, $\mathrm{Q}_{\mathrm{s}}$, and effects of $\mathrm{RF}$ loops. The longitudinal diagnostics are particularly important in light of the expected Dual Harmonic RF upgrade [5], which is designed to increase intensity by $50 \%$. Phase space distributions and dynamics may be reconstructed from longitudinal profiles using 2D tomographic techniques [6]. Comparisons with results from space charge codes are also planned.

\section{CONCLUSIONS}

Upgrades to the diagnostics are providing much valuable and detailed information on most aspects of beam dynamics. The challenge now lies in making full use of low and high intensity measurements to understand and optimise high intensity performance.

\section{REFERENCES}

[1] Development of the ISIS Synchrotron Diagnostics, D J Adams et al, Proc. EPAC 98.

[2] Spallation Neutron Source, B Boardman, RAL Report RL-82-006.

[3] Low intensity and Injection Studies on the ISIS Synchrotron, C M Warsop, Proc. ICANS XII, RAL Report RAL 94-025.

[4] Circular Accelerators and Storage Rings, P Bryant, K Johnsen.

[5] A Possible Upgrade for ISIS, M Harold et al, Proc. PAC 97.

[6] Tomographic Measurements of Longitudinal Phase Space Density, S Hancock et al, Proc. EPAC 98 